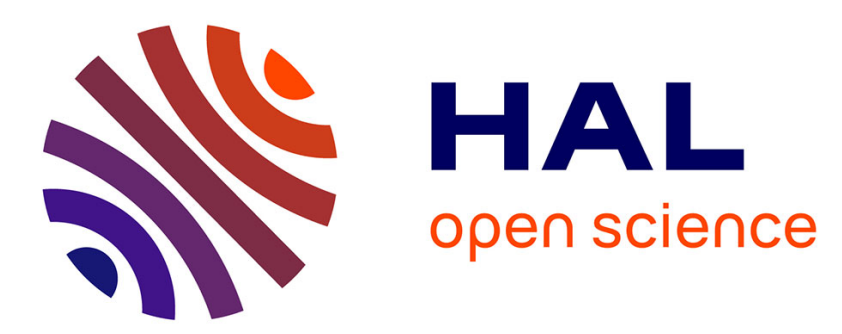

\title{
Quand Mauboussin affiche ses prix en 4 par 3 dans le métro - Retour aux fondamentaux du marketing de masse dans le secteur du luxe
}

Thierry Delécolle, Béatrice Parguel

\section{- To cite this version:}

Thierry Delécolle, Béatrice Parguel. Quand Mauboussin affiche ses prix en 4 par 3 dans le métro Retour aux fondamentaux du marketing de masse dans le secteur du luxe. Décisions Marketing, 2010, 59, pp.79-82. halshs-00634447v2

\section{HAL Id: halshs-00634447 \\ https://shs.hal.science/halshs-00634447v2}

Submitted on 9 Aug 2012

HAL is a multi-disciplinary open access archive for the deposit and dissemination of scientific research documents, whether they are published or not. The documents may come from teaching and research institutions in France or abroad, or from public or private research centers.
L'archive ouverte pluridisciplinaire HAL, est destinée au dépôt et à la diffusion de documents scientifiques de niveau recherche, publiés ou non, émanant des établissements d'enseignement et de recherche français ou étrangers, des laboratoires publics ou privés. 


\section{Retour aux fondamentaux du marketing de masse dans le secteur du luxe}

\section{Quand Mauboussin affiche ses prix en 4 par 3 dans le métro Entretien avec Alain Nemarq ${ }^{1}$}

Thierry Delécolle

Docteur en Sciences de Gestion de l'Université Paris-Dauphine

Coordonnées personnelles :

Tél. 0660453523

tdelecolle@gmail.com

Béatrice Parguel

Docteur en Sciences de Gestion de l'Université Paris-Dauphine

Coordonnées personnelles :

Tél. 0607601892

beatrice.parguel@gmail.com

\footnotetext{
${ }^{1}$ Alain Nemarq est le Président de Mauboussin. Cet entretien a été réalisé par Thierry Delécolle et Béatrice Parguel, tous deux Docteurs de Dauphine - Recherches en Management (CNRS UMR 7088).
} 


\title{
Retour aux fondamentaux du marketing de masse dans le secteur du luxe
}

\section{Quand Mauboussin affiche ses prix en 4 par 3 dans le métro Entretien avec Alain Nemarq}

\section{Résumé}

Vieille de plus de 180 ans, la joaillerie Mauboussin fait figure d'enfant terrible sur la Place Vendôme depuis que Dominique Frémont en a pris le contrôle il y a une dizaine d'années. Alain Nemarq, son Président, revient sur la stratégie marketing qu'il a mise en place depuis son arrivée en 2002. Il détaille ainsi le nouveau modèle de croissance de la Maison et décrypte les campagnes de communication qui lui ont récemment valu d'être taxé de "casseur de codes" sur le marché de la haute joaillerie. Il explique par ailleurs en quoi le fait d'être "un nain" sur ce marché représente à ses yeux un atout considérable.

Mots-clés : marketing du luxe, marketing de masse, communication sociétale, cas d'école

\section{Return to mass marketing fundamentals in the luxury sector When Mauboussin shows its prices on subway billboards Interview with Alain Nemarq}

\begin{abstract}
:
Over 180 years old, the Mauboussin jeweler's shop looks like a rebellious child on the Place Vendôme (Paris, France) since Dominique Frémont took over almost ten years ago. Alain Nemarq, the President, explains the marketing strategy he has used since his arrival in 2002. He also details the new growth model and gives us the insights to understand the advertising campaigns which recently earned him the title of "rule breaker" on the market of luxury jewelery. Besides, he tells us that the fact of being "a dwarf" on this market represents in his eyes a huge asset.
\end{abstract}

Keywords: luxury marketing, mass marketing, societal communication, case study 
DM : Comment se positionne Mauboussin sur le marché de la haute joaillerie?

Mauboussin, c'est 42 millions d'euros de CA sur le marché de la joaillerie dont 15 sur le segment de la haute joaillerie, qui concerne les pièces de plus de 7.000 euros et atteint grosso modo avant la crise les 700 millions d'euros en valeur retail. Sur ce segment, Mauboussin est donc un nain. Au-delà de la France, qui représente $60 \%$ des ventes, Mauboussin est également implanté en Belgique, en Suisse et en Russie, et a plus récemment ouvert des boutiques au Japon, à Singapour, à Hong Kong, à Macao, en Corée et aux Etats-Unis.

DM : Quel est le rôle de ces nouvelles implantations dans votre stratégie?

En 2002, lorsque Dominique Frémont a pris le contrôle de l'entreprise, il m'a fixé comme feuille de route de faire passer le CA de la Maison de 12 à 100 millions d'euros à l'horizon 2014. Cela passe très concrètement par l'augmentation de la surface commerciale de 300 à $4.000 \mathrm{~m}^{2}$ sur cette même période.

DM : L'international est donc un levier de croissance essentiel. Au-delà, pouvez-vous nous en dire plus sur votre stratégie marketing?

Né en 1827, Mauboussin est une vieille maison qui présente la particularité d'avoir toujours été axée sur la création. Pendant longtemps, Mauboussin s'est contenté de se développer à partir d'une clientèle uniquement constituée de happy few. En cela, la Maison respectait parfaitement les lois du marketing en vigueur dans le secteur du luxe. Mais en 1998, la perte de son principal client, le Sultan de Brunei, qui représentait alors $80 \%$ de son activité, l'a poussée à redéfinir sa stratégie. La création est restée au service de l'émotion, mais la Maison s'est tournée vers une nouvelle cible, celle de la femme moderne.

\section{DM : Comment définiriez-vous cette femme?}

Cette femme, c'est une femme de l'époque. C'est une femme qui a su gagner sa liberté au cours de la deuxième partie du $\mathrm{XX}^{\text {ème }}$ siècle et qui assume désormais sa position sociale. Rappelons-le, avant 1963, les femmes n'avaient pas le droit d'avoir un chéquier et avant 1966, elles ne pouvaient pas gérer leurs biens ou choisir une profession sans l'autorisation de leur mari. Ce n'est qu'en 1971 qu'on a ouvert les grandes écoles aux 
filles ; jusque là, les garçons avaient vocation à être des managers et les filles à être leur secrétaire de direction. A cette époque, le bijou était essentiellement acheté par un homme pour sa femme ou sa compagne. Il pouvait lui apparaitre comme un placement financier, mais aussi comme un signal de son statut social et de son pouvoir d'achat. L'achat d'un bijou était donc extrêmement impliquant pour l'homme. Je dis souvent avec humour que le bijou était un trophée offert à un autre trophée, c'était ça la société française de l'époque... Maintenant, ma cible à moi, c'est la femme d'aujourd'hui, quels que soient son âge ou son pouvoir d'achat. La seule chose qui m'importe, c'est que ces femmes d'aujourd'hui assument leurs responsabilités et que mes créations leur apportent des émotions.

DM : Récemment, vous avez ouvertement affiché le prix de certains de vos produits dans les grands médias - sur TF1, dans le métro ou dans la presse magazine - et la presse vous a qualifié de «casseur de codes»du luxe. Pourriez-vous nous aider à décrypter cette campagne de communication?

C'est vrai que j'ai brûlé certaines idées reçues en matière de communication, mais pas parce que j'avais envie de casser les codes, seulement parce que je m'adressais à une femme différente. Et en même temps, je suis aussi revenu aux fondamentaux du marketing. Vous connaissez la fameuse équation du marketing «faire connaître, faire aimer, faire acheter $\gg$ ?

Pour «faire connaître », je suis tous médias parce que la femme moderne, je veux la toucher pendant son temps de loisir lorsqu'elle feuillète des magazines de mode, mais aussi à d'autres moments où elle peut être touchée par la création, notamment durant ses déplacements, d'où l'affichage dans le métro, la publicité dans la presse quotidienne et également dans les journaux gratuits. Après, je communique plus fréquemment que mes concurrents, mais c'est simplement parce que je renouvelle mes collections trois fois plus rapidement.

Pour «faire aimer », je suis parti de l'idée que le porte manteau ne serait jamais aussi seyant que le manteau porté par une personne qui va le sublimer. Ce qui constitue une révolution en matière de création publicitaire, c'est qu'à partir du moment où je voulais toucher toutes les femmes, il fallait que le mannequin qui présente l'objet reflète une 
image de la vraie vie, avec une vraie peau et une vraie pigmentation qui n'effacent pas totalement toutes les rides de la vie.

L'affichage des prix, c'est bien évidemment pour «faire acheter ». J'estime que la femme que je cible, qui est une femme que je respecte, est une femme intelligente et qu'elle a le droit de connaître le prix d'un objet sans avoir besoin de venir chez moi. Afficher les prix, cela réduit sa crainte de venir et cela lui donne la possibilité de venir essayer et de goûter ainsi à la création artistique. Et puis, moi je n'ai pas pour vocation de participer à un processus où l'homme qu'elle aime va lui offrir un bijou dont elle aura le sentiment qu'il aura coûté trois fois plus que sa valeur économique. Un bijou, ça vaut beaucoup plus que son prix, ça vaut la puissance de l'émotion qui a justifié l'achat. Sinon, ce ne serait qu'un échange de biens et dans ce cas, pourquoi offrir un bijou ? Autant offrir un chèque !

\section{DM : Qu'est ce qui vous permet d'afficher de tels tarifs?}

Vous savez, la société a beaucoup dérapé. Avec la période des golden boys au début des années 1980, on a connu une formidable inflation des prix, parce que l'idéologie dominante disait que pour qu'un produit soit de luxe, il fallait le rendre inaccessible. La hausse s'est ensuite amplifiée avec la mise en place de l'Euro. La cherté, c'est l'opium des riches, mais à un moment je me suis dit qu'il fallait arrêter de prendre les gens pour des imbéciles.

Du coup, et avec l'accord de l'actionnaire, j'ai baissé ma marge de l'ordre de $20 \%$ par rapport à la profession, de manière à ce que le prix des objets que je créais me paraisse plus justifié. Je trouvais que ça allait dans le sens d'une moralisation de l'histoire commerciale et je l'ai mis en œuvre. Après, je l'ai communiqué parce que cela aurait été complètement ridicule de le faire sans le communiquer.

DM : Au-delà de la communication commerciale, vous vous êtes également distingué par des « coups » plus ponctuels, comme l'opération «Yes we can! » (cf. encadré 1), la campagne du 14 juillet ou la commercialisation de vos produits sur ventesprivees.com. Pourriez-vous nous en dire plus?

Ce ne sont pas des coups, c'est que la marque Mauboussin se nourrit d'un double langage. Elle a ainsi une communication commerciale complètement centrée sur le 
produit, pour le «faire aimer», et en parallèle, elle a une communication sociétale. Je pense que dans un climat général de crise, le consommateur attend un peu d'optimisme et de générosité de la part des marques. Avec les campagnes «Yes we can!» ou «liberté, égalité, fraternité et l'amour alors ? , la marque Mauboussin prend le contrepied de la crise et cherche à transmettre, avec un peu de dérision, une autre vision de la société, plus optimiste et plus fraternelle. Dans ces messages, qui s'accompagnent toujours d'un cadeau, d'une remise, d'une invitation à partager un moment ou une gourmandise, la marque communique autrement parce qu'elle se veut un agent social d'espoir.

Du point de vue institutionnel, mes concurrents donnent plutôt dans le charity, mais c'est une formidable hypocrisie : en réalité, c'est un moyen d'écouler les stocks. A quoi bon se masquer? Quand je veux renouveler mes collections et que je dois nettoyer mes stocks, moi je vais sur ventesprivees.com et je l'assume.

DM : Cette stratégie en rupture avec celle de vos concurrents peut paraître risquée. Avez-vous des indicateurs pour nous permettre d'en apprécier la pertinence?

Moi, je suis quelqu'un de pragmatique. L'indicateur principal, c'est le taux de croissance. Mauboussin, à la fin du mois d'août, c'est $65 \%$ de croissance par rapport à l'an dernier. Notre stratégie nous a permis d'attirer une nouvelle clientèle. Quand j'ai pris la direction de l'entreprise, Mauboussin avait à peu près 3.000 clients en fichiers ; aujourd'hui nous en avons un peu plus de 100.000. Le risque aurait été de perdre notre clientèle traditionnelle, mais elle est restée. Et à côté de cela, on a pu recruter de nouveaux clients. Evidemment Mauboussin a désormais beaucoup plus de points de vente donc mathématiquement il y a plus de clients. Mais le recrutement dépasse notre politique d'ouverture de magasins. Vendôme par exemple est passé de 1.200 clients à 5.000 clients annuels à isopérimètre, cela vous donne une idée.

En ce qui concerne les indicateurs d'efficacité de nos opérations de communication, je ne dispose que de peu d'information. A priori, mes ventes de diamants sont très liées à la publicité que je fais à la télévision, mais au-delà je n'ai que des intuitions. On ne préteste pas nos campagnes et on ne réalise pas de post-tests non plus. En revanche, on gère suffisamment bien la relation client pour mesurer que notre stratégie a permis de développer la fidélité : on atteint désormais une moyenne de deux visites d'achat dans 
l'année. Avant, on offrait un bijou pour l'éternité et la visite chez un joailler était plutôt un évènement exceptionnel. Chez Mauboussin, on arrive à obtenir que l'achat d'une bague déclenche également celui du pendentif ou de la paire de boucles d'oreilles dans l'année, ce qui constitue une modification considérable du comportement d'achat. C'est un résultat important.

DM : Finalement, le fait d'être «un nain» est peut-être le premier atout de votre Maison?

Sa faiblesse et sa force, mais peut-être davantage sa force que sa faiblesse. C'est peutêtre une faiblesse pour l'actionnaire par rapport au retour sur investissement qu'il peut en espérer. Mais en termes de fonctionnement, c'est une formidable force. La réalité c'est que les petites maisons de création comme Mauboussin sont organisées autour du créateur, mais que celui-ci ne peut pas se contenter de créer les objets. C'est donc moi qui crée les collections, mais je gère aussi la communication pour ces objets : Jean Collette, un ami, crée les visuels et j'achète moi-même mes espaces publicitaires. Tout cela est rendu possible par la personnalité de l'actionnaire et la nature amicale de notre relation. Il me laisse tout piloter, mais il constitue en même temps un fort contrepouvoir et n'hésite pas à me dire quand ce n'est pas possible et qu'il faut s'arrêter. Pour le reste, le siège social de Mauboussin, c'est 23 personnes. Tous les gens qui travaillent ici sont soit des gens que j'ai moi-même recrutés ou qui étaient déjà des fidèles de la Maison quand je suis arrivé, et désormais on forme une véritable famille.

DM : Quel est l'impact de la crise actuelle sur l'efficacité de votre stratégie? Quels seront vos prochains relais de croissance?

Savoir si une baisse du marché du luxe de 20 ou $25 \%$ peut nous influencer? Parce que je ne pèse rien sur ce marché, je ne pense pas que la crise peut nous impacter. Et puis, il faut s'entendre sur ce qu'on appelle «la crise ». Ce n'est pas simplement une crise financière qui a entraîné une crise économique, c'est aussi une crise identitaire et un tournant sociologique important. Je pense qu'on va sortir de la course à la fausse sophistication et que ma stratégie me placera en bonne position si je continue à créer des bijoux qui sont au goût des femmes. 
Pour ce qui est des leviers de croissance, le principal, c'est ma surface commerciale, comme je vous le disais précédemment. C'est $2.100 \mathrm{~m}^{2}$ aujourd'hui, $4.000 \mathrm{~m}^{2}$ en 2014. Pour l'instant, un $\mathrm{m}^{2}$ nous coûte grosso modo 2.500 euros d'aménagement, 5.000 euros de stocks et nous rapporte en moyenne un CA de 20.000 euros avec 13.000 euros de marge brute et 3.200 euros de publicité. Tout cela fait un CA de 250 euros par personne utile touchée par un point de vente. La vraie question, c'est de savoir s'il n'y a pas un système où le $\mathrm{CA}$ au contact utile pourrait être plus faible et où je n'assumerais plus la masse d'investissement que représentent mes ouvertures de magasins. Le Web par exemple, faut-il aller vers un Web marchand pour tenter d'accélérer la croissance ? Pour l'instant, je manque encore d'information. Avant d'aller sur le Web, il faut savoir ce qu'on a envie d'y faire ou d'y dire et savoir comment les femmes vivent Internet ou comment on a envie d'y communiquer avec elles. Je pense que la femme que je décrivais tout à l'heure n'est pas encore suffisamment libérée de ses contraintes pour être en consommation de création sur Internet, mais elle peut le devenir demain... 


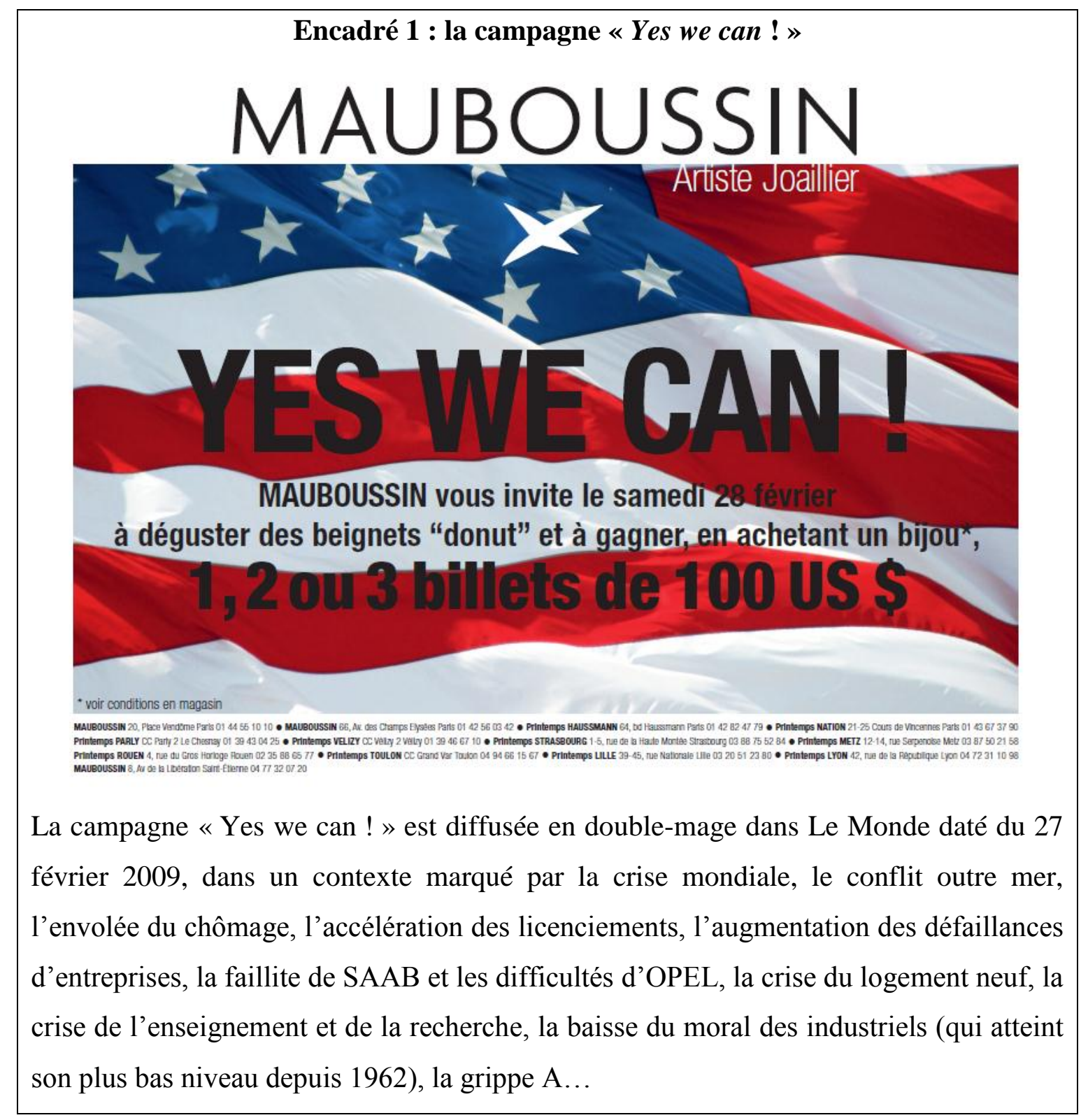

